\title{
REMOVABLE SETS FOR POINTWISE SOLUTIONS OF ELLIPTIC PARTIAL DIFFERENTIAL EQUATIONS $\left.{ }^{(1}\right)$
}

\author{
BY \\ JIM DIEDERICH( $\left.{ }^{2}\right)$
}

\begin{abstract}
We prove that dense sets of zero newtonian capacity are removable for
\end{abstract} bounded generalized pointwise solutions of second order elliptic equations.

0. Introduction. A classical theorem for harmonic functions in $K$-space is that every closed set $E$ of zero newtonian capacity is removable for the class of bounded harmonic functions. J. Serrin [14] has generalized this theorem to bounded solutions of linear second order elliptic partial differential equations. Our aim is to remove dense sets, i.e. $F_{\sigma}$ sets, without requiring any information about the functions on the exceptional set. This necessitates introducing a notion of generalized pointwise solutions which leads to a regularity theorem (Theorem 1) for a class of elliptic equations for which the adjoint cannot necessarily be defined. This work is an extension of some of the concepts developed by V. L. Shapiro in [17]. Our theorems and more specific comments are to be found in the next section.

1. Preliminaries. We shall work in $K$-dimensional Euclidean space, $E_{K}, K \geqq 3$, and shall use the following notation: $x=\left(x_{1}, \ldots, x_{K}\right)$ and $B(x, r)=$ the open $K$-ball with center $x$ and radius $r . \Omega$ will always denote an open, bounded set in $E_{K} \cdot|E|$ will denote the measure of $E$ and $\operatorname{Cap}(E)$ the $K$-dimensional newtonian capacity, see [3, p. 16]. All functions shall have the extended reals for their range.

A function $u$ defined on $\Omega$ is said to be in $t_{\alpha}\left(x_{0}\right), \alpha \geqq 0$, if $u$ is in $L_{1}$ in a neighborhood of $x_{0}$, and if there is a polynomial $P(y)$ of degree $\leqq \alpha$ such that

$$
|B(0, r)|^{-1} \int_{B(0, r)}\left|u\left(x_{0}+y\right)-P(y)\right| d y=o\left(r^{\alpha}\right) \quad \text { as } r \rightarrow 0 .
$$

For $u$ in $t_{2}\left(x_{0}\right)$, we define the generalized partials $D_{i} u\left(x_{0}\right), D_{i j} u\left(x_{0}\right)$ to be the coefficients of $y_{i}, y_{i} y_{j} / 2$ ! in $P(y)$ respectively. (The class $t_{\alpha}$ was defined in [2] and since has appeared in a number of places in the literature, in particular in [17].)

Presented to the Society, January 21, 1971 under the title Removable sets for pointwise solutions of elliptic equations; received by the editors October 19, 1970 and, in revised form, June 28, 1971.

AMS 1969 subject classifications. Primary 3542; Secondary 3524.

Key words and phrases. Removable sets, capacity, regularity, generalized partials, generalized subelliptic operator, subelliptic, subsolution.

(1) This paper is based on the author's thesis, submitted at the University of California at Riverside. The author wishes to acknowledge his indebtedness to Professor V. L. Shapiro, under whose direction the thesis was written.

$\left.{ }^{2}\right)$ NDEA Fellow. 
Throughout we shall consider linear second order elliptic partial differential equations of the form (the summation convention is adopted)

$$
L u=a_{i j}(x) u_{i j}+b_{i}(x) u_{i}+c(x) u=0 \quad(c(x) \leqq 0) .
$$

We also require that the coefficients of the operator $L$ be $\alpha$-Hölder continuous for some $0<\alpha \leqq 1$, with Hölder constant $K_{1}$. In addition $L$ is uniformly elliptic, i.e. for any nonzero vector $\xi$ and any $x$ in $\Omega$

$$
K_{2}|\xi|^{2} \leqq a_{i j}(x) \xi_{i} \xi_{j} \leqq K_{2}^{-1}|\xi|^{2} .
$$

With such restrictions on $L$, the maximum principle and the solution of the Dirichlet problem hold. Furthermore we normalize the trace $\sum a_{i i}(x)=K$ for $x$ in $\Omega$; since the trace is invariant under similarity transformation, the sum of the eigenvalues of the matrix $\left(a_{i j}(x)\right)$ must be $K$. This is simply for convenience in establishing Lemma 5.

For $u$ in $t_{2}\left(x_{0}\right)$, we say that $u$ is a $t_{2}$ solution of (2) at $x_{0}$ if

$$
a_{i j}\left(x_{0}\right) D_{i j} u\left(x_{0}\right)+b_{i}\left(x_{0}\right) D_{i} u\left(x_{0}\right)+c\left(x_{0}\right) u\left(x_{0}\right)=0 .
$$

We are now ready to state two of our theorems. Theorem 1 is a regularity theorem for $t_{2}$ solutions of (2) and is used to establish Theorem 3 on removable $F_{\sigma}$ sets for (2). Theorem 3 is a generalization of Serrin's Theorem 1 [14] for $L_{\infty}$ solutions and sets of capacity zero. The generalization of Carleson's Theorem 2 [3] for Lip $\alpha$ solutions of (2) is handled in a paper by Shapiro and Diederich in which additional restrictions are placed on the coefficients of $L$.

THEOREM 1. Let $u$ be a $t_{2}$ solution of (2) at each point of $\Omega$ and let $u$ be in $L_{\infty}$. Then $u$ is a classical solution of (2) in the sense that $u$ is in $C^{2+\alpha}$ and $L u=0$.

THEOREM 3. Let $u$ be a $t_{2}$ solution of (2) in $\Omega-E$, where $E$ is an $F_{\sigma}$ set, and let $u$ be in $L_{\infty}$. Then a necessary and sufficient condition that $E$ be removable, i.e. that $u$ is equal a.e. in $\Omega$ to a classical solution $v$ of $(2)$, is that $\operatorname{Cap}(E)=0$.

A few remarks on the above theorems are in order. First, the hypotheses do not require that the function be defined on the exceptional set $E$. Second, the exceptional set is allowed to be dense in $\Omega$. (Neither the Looman-Menchoff Theorem [13, p. 199] nor Serrin's Theorem 1 [14, p. 68] satisfy both of these requirements.) Clearly, then, the classical notion of derivative cannot be employed and the global nature of weak (i.e. distribution) derivative disallows its use. Arsove, in [1, p. 98], allows the exceptional set to be dense and thereby uses the generalized Laplacian (see $\$ 2$ for the definition) for his pointwise definition of operator. However, the hypothesis of continuity cannot be eliminated (see the discussion which occurs at the end of the Appendix) for this generalized operator. Hence we use the stronger notion of $t_{2}$ derivative. Also note that the notion of weak solution does not apply in general to our class of operators. Briefly, Theorem 2 is a generalization of Arsove's Theorem 6 [1, p. 98] on subharmonic functions, to functions which are subelliptic. This theorem is also used in establishing Theorem 3. 
2. Generalized derivatives. Several important properties of differentiable functions carry over to functions in $t_{\alpha}$. For $f$ and $g$ in $t_{1}\left(x_{0}\right)$, the following are easily verified: (a) $f+g$ is in $t_{1}\left(x_{0}\right)$, (b) if $g$ is in $L_{\infty}$, then $f \cdot g$ is in $t_{1}\left(x_{0}\right)$, and (c) if $T$ is a 1-1 linear transformation on $E_{K}$, then $f \circ T$ is in $t_{1}\left(T^{-1} x_{0}\right)$ where the $t_{1}$ partials of $f+g, f \cdot g$, and $f \circ T$ are given canonically in terms of the $t_{1}$ partials of $f, g$, and $T$. It is also clear that if $f$ is in $t_{1}\left(x_{0}\right)$ and $f\left(x_{0}\right)$ is a local maximum, then $D_{i} f\left(x_{0}\right)=0$, for $i=1,2, \ldots, K$. In $E_{1}$, differentiability of $f$ at $x_{0}$ ensures continuity of $f$ at $x_{0}$; similarly, $f$ in $t_{1}\left(x_{0}\right)$, even in $E_{K}$, ensures the mean continuity of $f$ at $x_{0}$ as can be seen from (1).

Global behavior is quite different, even in $E_{1}$. If $f^{\prime}$ and $f^{\prime \prime}$ exist in an interval $I$, then $f$ and $f^{\prime}$ are continuous and bounded on compact subsets of $I$. But $f$ in $t_{2}(x)$, for all $x$ in $I$, does not guarantee that $D_{1} f(x)$ is even in $L_{1}$ on compact subsets of $I$. A simple example is $f(x)=x^{3} \sin x^{-3}$ on $(-1,1)$, where $D_{1} f(0)=D_{11} f(0)=0$. Indeed, the $t_{\alpha}$ notion remains simply a pointwise generalization of differentiation. (Note that $(x-t)^{3} \sin (x-t)^{-3}$ is a continuous $t_{2}$ solution of the wave equation in the plane and is not a classical, i.e. $C^{2}$, solution.)

We now consider pointwise generalizations of differentiation which are implied by the $t_{\alpha}$ notion. For $f$ in $t_{1}\left(x_{0}\right)$ we observe from (1) that

$$
\begin{aligned}
\left(r^{2}|B(0, r)|\right)^{-1} & \left|\int_{B(0, r)} f\left(x_{0}+y\right) y_{i} d y-(K+2)^{-1} r^{2}\right| B(0, r)\left|D_{i} f\left(x_{0}\right)\right| \\
& \leqq(r|B(0, r)|)^{-1} \int_{B(0, r)}\left|f\left(x_{0}+y\right)-P(y)\right| d y=o(1) .
\end{aligned}
$$

Hence

$$
\begin{aligned}
\lim _{r \rightarrow 0} d_{i}(r) f\left(x_{0}\right) & =\lim _{r \rightarrow 0}(K+2)\left(r^{2}|B(0, r)|\right)^{-1} \int_{B(0, r)} f\left(x_{0}+y\right) y_{i} d y \\
& =D_{i} f\left(x_{0}\right)
\end{aligned}
$$

Likewise for $f$ in $t_{2}\left(x_{0}\right)$ we see from (1) that

$$
\lim _{r \rightarrow 0} 2(K+2)\left(r^{2}|B(0, r)|\right)^{-1} \int_{B(0, r)} f\left(x_{0}+y\right)-f\left(x_{0}\right) d y=\sum D_{i i} f\left(x_{0}\right),
$$

which is the classical notion of generalized Laplacian.

We define the upper generalized elliptic operator $L^{*}$ as follows: to each point $x_{0}$ in $\Omega$ associate the matrices $\left(a_{i j}\left(x_{0}\right)\right)$ and $\left(\lambda_{i}\left(x_{0}\right)\right)$, the latter being the matrix of eigenvalues of $\left(a_{i j}\left(x_{0}\right)\right)$. Since the eigenvalues are positive, consider the ellipse centered at the origin with axes $\lambda_{1}^{1 / 2}\left(x_{0}\right), \ldots, \lambda_{K}^{1 / 2}\left(x_{0}\right)$. Denote the rotation of this ellipse by the transformation $\mathscr{P}$, where $\mathscr{P} *\left(a_{i j}\left(x_{0}\right)\right) \mathscr{P}=\left(\lambda_{i}\left(x_{0}\right)\right)$, by $\operatorname{El}\left(x_{0}: 0,1\right)$. Denote the homothetic transformation $r$ of $\mathrm{El}\left(x_{0}: 0,1\right)$ by $\mathrm{El}\left(x_{0}: 0, r\right)$.

Set for $f$ in $L_{1}$,

$$
L^{\prime}(r) f\left(x_{0}\right)=2(K+2)\left(r^{2}\left|\mathrm{El}\left(x_{0}: 0, r\right)\right|\right)^{-1} \int_{E 1\left(x_{0}: 0, r\right)} f\left(x_{0}+y\right)-f\left(x_{0}\right) d y
$$


Then define $L^{*} f\left(x_{0}\right)$ to be

$$
\limsup _{r \rightarrow 0}\left[L^{\prime}(r) f\left(x_{0}\right)+\sum b_{i}\left(x_{0}\right) d_{i}(\delta r) f\left(x_{0}\right)+c\left(x_{0}\right) f\left(x_{0}\right)\right]
$$

where $\delta$ allows $B(0, \delta r) \subseteq \mathrm{El}\left(x_{0}: 0, r\right)$ for each $x_{0}$. For $L_{*} f\left(x_{0}\right)$ we use the lim inf. Note for $f$ in $t_{2}\left(x_{0}\right)$ that $L^{*} f\left(x_{0}\right)=L_{*} f\left(x_{0}\right)=$ left side of (4).

A function $v$ is called subelliptic $\left({ }^{3}\right)$ in a region $\Omega$ in $E_{K}$ if (1) $v$ is upper semicontinuous and (2) for each subdomain $G$, if $L u=0$ in $G$ and $\lim \sup _{x \rightarrow y: x \in G} v(x)$ $\leqq u(y)$, for every $y$ in $\partial G$, then $v(x) \leqq u(x)$ in $G$.

THEOREM 2. Let $u$ be a continuous real-valued function on $\Omega$ such that $L_{*} u(x)$ $>-\infty$ holds except on an $F_{\sigma}$ set $E$ with $\operatorname{Cap}(E)=0$. Let $L_{*} u(x) \geqq 0$ hold almost everywhere. Then $u$ is subelliptic on $\Omega$.

We should mention here that the notions of generalized derivatives and operators have been heavily exploited in the work of Verblunsky, Shapiro [15], [16], [17], Philipp [9], and others to establish uniqueness results in the theory of trigonometric series and partial differential equations.

3. Regularity of $t_{2}$ solutions. We shall need some definitions and lemmas in order to establish Theorem 1. $T_{K}=\left\{x:-\pi<x_{j} \leqq \pi, j=1, \ldots, K\right\} ; m$ will denote an integral lattice point; for a function $f$ in $L_{1}\left(T_{K}\right)$ we shall set

$$
\hat{f}(m)=(2 \pi)^{-K} \int_{T_{K}} f(x) e^{-i(m, x)} d x .
$$

Given $f$ in $L_{1}\left(T_{K}\right)$, we shall say that $f$ is extended by periodicity to all of $E_{K}$ if $f$ is defined in all of $E_{K}$ and is $2 \pi$-periodic in each variable. By $A_{t}(f, x)$ we denote the Abel sum of $f, \sum_{m} \hat{f}(m) e^{i(m, x)-|m| t}$, for $x$ in $E_{K}$ and $t>0$, which also has the integral representation

$$
b_{K} t \int_{E_{K}} f(y)\left[t^{2}+|x-y|^{2}\right]^{-(K+1) / 2} d y
$$

where $b_{K}$ is a dimensional constant (see [15]).

LEMMA 1. Let $f$ be in $L_{1}\left(T_{K}\right)$ and extended by periodicity to $E_{K}$. In addition, let $f$ be in $t_{2}\left(x_{0}\right)$ with generalized partials $D_{i} f\left(x_{0}\right)$ and $D_{i j} f\left(x_{0}\right)$. Then

$$
\lim _{t \rightarrow 0}\left(\partial / \partial x_{i}\right) A_{t}\left(f, x_{0}\right)=D_{i} f\left(x_{0}\right)
$$

and

$$
\lim _{t \rightarrow 0}\left(\partial^{2} / \partial x_{i} \partial x_{j}\right) A_{t}\left(f, x_{0}\right)=D_{i j} f\left(x_{0}\right)
$$

$\left({ }^{3}\right)$ The terminology "subsolution" or "subfunction" is standard. "Subelliptic" is used here in conjunction with the upper generalized elliptic operator. 
Proof. We establish the lemma for (5) with $i \neq j$ and $x_{0}=0$ without loss. For $t>0$, the second partial of $A_{t}(f, x)$ at 0 is

$$
t b_{K}(K+1)(K+3) \int_{E_{K}} f(y) y_{i} y_{j}\left[t^{2}+|y|^{2}\right]^{-(K+5) / 2} d y .
$$

Let $P_{1}(x)$ be the $t_{2}$ total differential of $f$ at 0 , and let $\lambda(x)$ be a $C^{\infty}$ function which is identically 1 on $B(0,1), 0$ on $B(0, \pi / 2)^{C}$, and between 0 and 1 otherwise. Set $P(x)=P_{1}(x) \lambda(x)$, periodized to $E_{K}$. Then

$$
\left(\partial^{2} / \partial x_{i} \partial x_{j}\right) A_{t}(f, x)=\left(\partial^{2} / \partial x_{i} \partial x_{j}\right)\left[A_{t}(f-P, x)+A_{t}(P, x)\right] .
$$

Clearly $\lim _{t \rightarrow 0}\left(\partial^{2} / \partial x_{i} \partial x_{j}\right) A_{t}(P, x)_{x=0}=D_{i j} f(0)$. We need only show that

$$
\lim _{t \rightarrow 0}|I(t)|=0 \text {, }
$$

where $I(t)=\left(\partial^{2} / \partial x_{i} \partial x_{j}\right) A_{t}(f-P, x)_{x=0}$. Let $\varepsilon>0$, and select $r_{0}$ such that for $r \leqq r_{0}$

$$
F(r)=\int_{B(0, r)}|f(y)-P(y)| d y \leqq \varepsilon r^{2}|B(0, r)| .
$$

We now proceed as in $[15$, p. 57]:

$$
|I(t)| \leqq C(K) t \int_{0}^{r_{0}} r^{2}\left[t^{2}+r^{2}\right]^{-(K+5) / 2} d F(r)+C(K) t \int_{r_{0}}^{\infty} r^{2}\left[t^{2}+r^{2}\right]^{-(K+5) / 2} d F(r)
$$

the second term is $o(1)$. Integrating by parts we get

$$
\begin{aligned}
|I(t)| & \leqq C(K) t \int_{0}^{r_{0}} r F(r)\left[t^{2}+r^{2}\right]^{-(K+5) / 2} d r+o(1) \\
& \leqq \varepsilon \cdot C(K) t \int_{0}^{r_{0}} r^{K+1}\left[t^{2}+r^{2}\right]^{-(K+3) / 2} d r+o(1) \\
& \leqq \varepsilon \cdot C(K)+o(1)
\end{aligned}
$$

after using the transformations $r=t s$ and $s=\tan \theta$, completing the proof.

LEMma 2. Let $f$ be in $t_{2}(x)$ for all $x$ in $\Omega$. Then for each open ball $B \subseteq \Omega$, there is an open subball $B_{1} \subset B$ in which $f$ is continuous $\left({ }^{4}\right)$.

Proof. Without loss, $\Omega \subset T_{K}$ and $B \subset B(0,1)$. Redefine $f(x)$ to be $f(x) \lambda(x)$ where $\lambda$ is the same as in Lemma 1. For $t>0$, denote the Laplacian applied to $A_{t}(f, x)$ by $\Delta A_{t}(f, x)$. Then $\Delta A_{t}(f, x)=-\sum_{m \neq 0}|m|^{2} \hat{f}(m) e^{i(m, x)-|m| t}$ converges to $\sum D_{i i} f(x)$ as $t \rightarrow 0$ by Lemma 1 . Following [15, p. 67] we see that $-\sum_{m \neq 0}|m| \hat{f}(m) e^{i(m, x)-|m| t}$ and $-\sum_{m \neq 0} \hat{f}(m) e^{i(m, x)-|m| t}$ converge. By the continuity of $\Delta A_{t}(f, x)$ in $x$ and $t$ and the periodicity in $x$, there is a sequence $t_{1}>t_{2}>\cdots>t_{i}>\cdots$ such that $t_{i} \rightarrow 0$ and

$$
\sup _{x \operatorname{in} E_{K}}\left\|\Delta A_{t}(f, x)-\Delta A_{t_{i}}(f, x)\right\| \leqq 1 \quad \text { for } t_{i+1} \leqq t \leqq t_{i} .
$$

$\left.{ }^{4}\right) B$ can also be a closed set; $f$ will then be continuous in $B_{1} \cap B$ in the relative topology. 
The Baire category theorem yields a subball $B_{1} \subset B$ on which $\Delta A_{t_{i}}(f, x)$ is uniformly bounded for $i=1,2, \ldots$ and thus by (6) for $t>0$. Hence $A_{t}(f, x)$ converges uniformly in $B_{1}$. Since $f$ is mean continuous in $\Omega$, by Theorem $2\left[15\right.$, p. 55] $A_{t}(f, x)$ converges to $f$ in $B(0,1)$. So $f$ is continuous in $B_{1}$ completing the proof.

Lemma 3. Let $u$ be continuous in $\Omega$ and let $L^{*} u(x) \geqq 0$ for $x$ in $\Omega$. Then $u$ is subelliptic in $\Omega$.

Proof. Let $\bar{B}$ be a closed ball in $\Omega$. Let $v$ be the solution of (2) in $B$ with boundary values $u$. Select $h_{n}$ such that $L h_{n}(x) \equiv 1 / n$, for $x$ in $B$, and $h_{n} \equiv 0$ on $\partial B$. We first show that $u_{n}(x)=u(x)+h_{n}(x) \leqq v(x)$ in $\bar{B}$. If $v$ does not majorize $u_{n}$, then by the continuity of $u$ there is a point $x_{0}$ in $B$ at which $u_{n}-v$ takes its positive maximum.

If we show that

$$
0 \geqq L^{*}\left(u_{n}-v\right)\left(x_{0}\right)
$$

then, setting $u_{n}-v=U_{n}$,

$$
0 \geqq L^{*} U_{n}\left(x_{0}\right)=L^{*} u_{n}\left(x_{0}\right)=L^{*} u\left(x_{0}\right)+L h_{n}\left(x_{0}\right) \geqq 1 / n ;
$$

thus $u_{n} \leqq v$ in $\bar{B}$.

We assume that $b_{i}\left(x_{0}\right) \geqq 0$ and prove (7). Since $U_{n}\left(x_{0}\right)>0$ and $c\left(x_{0}\right) \leqq 0$, $c\left(x_{0}\right) U_{n}\left(x_{0}\right) \leqq 0$. Also

$$
\begin{aligned}
L^{\prime}(r) U_{n}\left(x_{0}\right)+ & \sum b_{i}\left(x_{0}\right) d_{i}(\delta r) U_{n}\left(x_{0}\right) \\
= & 2(K+2)\left[r^{2}\left|\operatorname{El}\left(x_{0}: 0, r\right)\right|\right]^{-1} \int_{E 1\left(x_{0}: 0, r\right)} U_{n}\left(x_{0}+y\right)-U_{n}\left(x_{0}\right) d y \\
& +\sum(K+2)\left[(\delta r)^{2}|B(0, \delta r)|\right]^{-1} b_{i}\left(x_{0}\right) \\
& \cdot\left\{\int_{B(0, \delta r): y_{i} \geqq 0}+\int_{B(0, \delta r): y_{i} \leqq 0}\left[U_{n}\left(x_{0}+y\right)-U_{n}\left(x_{0}\right)\right] y_{i} d y\right\}
\end{aligned}
$$

which is nonpositive for $r$, hence $\left|y_{i}\right|$, satisfying

$$
K r \delta^{-(K+2)} \max _{i}\left\{b_{i}\left(x_{0}\right)\right\}|B(0, r)|^{-1} \leqq 2\left|\mathrm{El}\left(x_{0}: 0, r\right)\right|^{-1}
$$

and (7) holds.

Since $h_{n} \equiv 0$ on $\partial B$, the maximum principle gives $h_{n}<h_{n+1}<\cdots<0$; also $h_{n} \rightarrow 0$ [7, p. 95], and therefore $u_{n}$ converges monotonically up to $u$. Consequently $u(x)=\lim _{n \rightarrow \infty} u_{n}(x) \leqq v(x)$ in $\bar{B}$. Thus $u$ is subelliptic and the proof is complete.

The following two theorems from the literature will be used in establishing the next lemma.

THEOREM a. For every $K$-ball $B\left(x_{0}, r\right)$ entirely in. $\Omega$, every $C^{2}$ solution of $\Delta u$ $=-\omega_{K} f$ in $B\left(x_{0}, r\right)$ satisfies the relation

$$
u\left(x_{0}\right)=\left|B\left(x_{0}, r\right)\right|^{-1} \int_{B\left(x_{0}, r\right)} u(x) d x-\int_{B\left(x_{0}, r\right)} \phi\left(r^{\prime}, r\right) f(x) d x
$$


where $f$ is continuous in $\bar{B}\left(x_{0}, r\right), \omega_{K}$ is the area of $\partial B(0,1)$, and

$$
\phi\left(r^{\prime}, r\right)=(K-2)^{-1}\left(r^{2-K}-\left(r^{\prime}\right)^{2-K}\right)+2^{-1} r^{2-K}\left(1-\left(r^{\prime}\right)^{2} r^{-2}\right),
$$

$r^{\prime}=\left|x-x_{0}\right|($ see $[4$, p. 277]).

TheOREM b (Schauder estimates). For each $C^{2}$ solution $u$ of $(2)$ in $B(0,1)$, the following interior estimates on the first and second derivatives of $u$ prevail in each subdomain $G$ having positive distance $d$ to $\partial B(0,1)$ :

$$
\max _{i, j: x \sin G}\left\{d\left|\left(\partial / \partial x_{i}\right) u(x)\right|, d^{2}\left|\left(\partial^{2} / \partial x_{i} \partial x_{j}\right) u(x)\right|\right\} \leqq C_{1}\|u\|_{\infty}
$$

where $C_{1}$ depends only on $K, K_{1}, K_{2}$, and $|B(0,1)|($ see $[4$, p. 335]).

LEMma 4 (APPROXIMATE MEAN VALUE). Let $u$ be a classical solution of (2) in a subregion of $\Omega$ which contains $\mathrm{El}\left(x_{0}: x_{0}, r\right)=\left\{x: x=x_{0}+y, y\right.$ in $\left.\mathrm{El}\left(x_{0}: 0, r\right)\right\}$. Then the following holds:

$$
\left.|| \mathrm{El}\left(x_{0}: 0, r / 2\right)\right|^{-1} \int_{\mathrm{El}\left(x_{0}: 0, r / 2\right)} u\left(x_{0}+y\right) d y-u\left(x_{0}\right) \mid \leqq C_{2}\|u\|_{\infty} r^{\alpha}
$$

where $C_{2}$ depends on $K, K_{1}, K_{2},\left\|b_{i}\right\|_{\infty},\|c\|_{\infty}$ and $\mathrm{El}\left(x_{0}: 0,1\right)$.

Proof. Since $L$ is elliptic we can assume that $a_{i j}\left(x_{0}\right)=\delta_{i j}$ and $x_{0}=0$, so El $(0: 0, r)=B(0, r)$. We define a new function $\tilde{u}$ and a new operator $\tilde{L}$ in $B(0,1)$ as follows:

(a) for $y$ in $B(0,1), \tilde{a}_{i j}(y), \tilde{b}_{i}(y)$, and $\tilde{c}(y)$ equal $a_{i j}(x), b_{i}(x)$, and $c(x)$ respectively, where $x=r y$.

(b) $\tilde{L} g(y)=\tilde{a}_{i j}(y) g_{i j}(y)+r \tilde{b}_{i}(y) g_{i}(y)+r^{2} \tilde{c}(y) g(y)$.

(c) $\tilde{u}(y)=u(x)$, where $x=r y$.

The constants $K_{1}$ and $K_{2}$ may be employed for the Hölder constant and uniform ellipticity constant of $\tilde{L}$ respectively;

$$
\begin{gathered}
\left|\tilde{a}_{i j}\left(y_{1}\right)-\tilde{a}_{i j}\left(y_{2}\right)\right| \leqq K_{1} r^{\alpha}\left|y_{1}-y_{2}\right|^{\alpha} \leqq K_{1}\left|y_{1}-y_{2}\right|^{\alpha} ; \\
K_{2}^{-1}|\xi|^{2} \geqq \tilde{a}_{i j}(y) \xi_{i} \xi_{j}=a_{i j}(x) \xi_{i} \xi_{j} \geqq K_{2}|\xi|^{2} .
\end{gathered}
$$

Clearly $\left(\partial^{2} / \partial y_{i} \partial y_{j}\right) \tilde{u}(y)=\left(\partial^{2} / \partial x_{i} \partial x_{j}\right) u(x) r^{2}$ and $\left(\partial / \partial y_{i}\right) \tilde{u}(y)=\left(\partial / \partial x_{i}\right) u(x) r$ so that $L u=0$ gives $\tilde{L} \tilde{u}=0$ in $B(0,1)$. From Theorem $\mathrm{b}$, (9),

$$
\begin{aligned}
\max _{i, j ; y \ln B(0,1 / 2)} & \left\{\frac{1}{2}\left|\left(\partial / \partial y_{i}\right) \tilde{u}(y)\right|,\left(\frac{1}{2}\right)^{2}\left|\left(\partial^{2} / \partial y_{i} \partial y_{j}\right) \tilde{u}(y)\right|\right\} \\
= & \max _{i, j ; x \operatorname{in} B(0, r / 2)}\left\{\frac{1}{2}\left|\left(\partial / \partial x_{i}\right) u(x)\right| r,\left(\frac{1}{2}\right)^{2}\left|\left(\partial^{2} / \partial x_{i} \partial x_{j}\right) u(x)\right| r^{2}\right\} \\
\leqq & C_{1}\|\tilde{u}\|_{\infty}=C_{1}\|u\|_{\infty} .
\end{aligned}
$$

In $B(0, r)$ we write

thus

$$
L u(x)=\Delta u(x)+\left(a_{i j}(x)-\delta_{i j}\right) u_{i j}(x)+b_{i}(x) u_{i}(x)+c(x) u(x)=0,
$$

$$
\Delta u(x)=-\left(a_{i j}(x)-\delta_{i j}\right) u_{i j}(x)-b_{i}(x) u_{i}(x)-c(x) u(x) \equiv f(x) .
$$


For $x$ in $B(0, r / 2)$, from (11) and the Hölder condition

$$
\begin{aligned}
|f(x)| & \leqq\left[K^{2} 4 K_{1} C_{1}\|u\|_{\infty} r^{-2+\alpha}+2 K\left\|b_{i}\right\|_{\infty} C_{1}\|u\|_{\infty} r^{-1}+\|c\|_{\infty}\|u\|_{\infty}\right] \\
& \leqq C_{2}^{\prime}\|u\|_{\infty} r^{-2+\alpha}, \quad r \leqq 1 .
\end{aligned}
$$

Using this in Theorem a, (8),

$$
\begin{aligned}
\left.|u(0)-| B(0, r / 2)\right|^{-1} \int_{B(0, r / 2)} u(x) d x \mid & \leqq C_{2}^{\prime}\|u\|_{\infty} r^{-2+\alpha} \int_{B(0, r / 2)}\left|\phi\left(r^{\prime}, r\right)\right| d x \\
& \leqq C_{2}\|u\|_{\infty} r^{-2+\alpha} r^{2} \leqq C_{2}\|u\|_{\infty} r^{\alpha},
\end{aligned}
$$

and the lemma is established.

Proof of Theorem 1. We first prove that $u$ is continuous. (This is accomplished by using Lemmas 1-4 in a fashion suggested by the methods in [15, pp. 65-78], which have been advanced in [17].) Assume, therefore, that the set of discontinuities $Z$ is not empty. Let $\bar{Z}$ be its closure in $\Omega$. By Lemma 2 we can find an open ball $B_{1}$ such that $B_{1} \cap Z \neq \varnothing$ and such that $u$ is continuous in $\bar{B}_{1} \cap \bar{Z}$ in the topology of $\bar{B}_{1} \cap \bar{Z}$.

For $x$ in $\bar{B}_{1} \cap \bar{Z}$ set

$$
U(x, r)=[r|\mathrm{El}(x: 0, r)|]^{-1} \int_{E 1(x: 0, r)}|u(x+y)-u(x)| d y .
$$

Clearly $U(x, r)$ is continuous in $\left(\bar{B}_{1} \cap \bar{Z}\right) \times(0,1]$. Also $U(x, r) \leqq M(x)$, a finite constant depending on $x$, since $u$ is in $t_{1}(x)$ for all $x$. Again, as in Lemma 2, we can find $r_{n}>r_{n+1}, r_{n} \rightarrow 0$, such that

$$
\sup _{x \operatorname{in} B_{1} \cap Z} \sup _{r_{n}+1 \leqq r \leqq r_{n}}\left|U(x, r)-U\left(x, r_{n}\right)\right| \leqq 1 .
$$

Thus by the Baire category theorem, there is a constant $M$ and an open ball $B_{2} \subset B_{1}$ such that $B_{2} \cap Z \neq \varnothing$ and

$$
U(x, r) \leqq M+1
$$

for $x$ in $\bar{B}_{2} \cap \bar{Z}$ and $0<r \leqq 1$.

We select $z_{0}$ in $B_{2} \cap Z$ and show that $u$ is indeed continuous at $z_{0}$, thus contradicting the assumption that $Z \neq \varnothing$. For $\varepsilon>0$, there is a $\delta<\varepsilon$ such that

(i) $B\left(z_{0}, \delta\right) \subset B_{2}$.

(ii) $3\left|u\left(z_{0}\right)-u(z)\right|<\varepsilon$ for $\left|z_{0}-z\right| \leqq \delta, z$ in $\bar{B}_{2} \cap \bar{Z}$, since $u$ is continuous in $\bar{B}_{1} \cap \bar{Z}$.

(iii) $3 r U(z, r)<\varepsilon\left(2 / K_{2}\right)^{-4 K}$ for $r \leqq \delta, z$ in $\bar{B}_{2} \cap \bar{Z}$, by (12) and the definition of $U(z, r)$.

(iv) $3 C_{2}\|u\|_{\infty} r^{\alpha}<\varepsilon$ for $r \leqq \delta$ for $C_{2}$ in Lemma 4 .

We need only show that for $x$ in $B\left(z_{0}, K_{2}^{3} \delta / 2\right),\left|u\left(z_{0}\right)-u(x)\right|<\varepsilon$. If $x$ is in $\bar{B}_{2} \cap \bar{Z}$ it follows from (ii). If $x$ is not in $\bar{Z}$, let $\mathrm{El}(x: x, \rho)$ be the largest ellipse about $x$ containing no points of $\bar{Z}$ in its interior $\left({ }^{5}\right)$. Clearly, by (3), $\rho \leqq K_{2}^{2} \delta / 2$ and

(5) $u$ is a classical solution in $\mathrm{El}(x: x, \rho)$; see the last paragraph of the proof for details. 
$\mathrm{El}(x: x, \rho) \subset B\left(z_{0}, \delta\right)$. Let $z^{*}$ be a point of $\bar{Z}$ and the boundary of $\mathrm{El}(x: x, \rho) ; z^{*}$ is in $\bar{B}\left(z_{0}, \delta\right) \subset \bar{B}_{2}$.

Thus

$$
\begin{aligned}
\left|u\left(z_{0}\right)-u(x)\right| \leqq & \left|u\left(z_{0}\right)-u\left(z^{*}\right)\right|+\left|u\left(z^{*}\right)-u(x)\right| \leqq \varepsilon / 3+\left|u\left(z^{*}\right)-u(x)\right| \text { by (ii), } \\
\leqq & \varepsilon / 3+\left.|u(x)-| \operatorname{El}(x: 0, \rho / 2)\right|^{-1} \int_{E 1(x: 0, \rho / 2)} u(x+y) d y \mid \\
& +\left.\left|u\left(z^{*}\right)-\right| \operatorname{El}(x: 0, \rho / 2)\right|^{-1} \int_{E 1(x: 0, \rho / 2)} u(x+y) d y \mid \\
\leqq & 2 \varepsilon / 3+\left.\left|u\left(z^{*}\right)-\right| \operatorname{El}(x: 0, \rho / 2)\right|^{-1} \int_{E 1(x: 0, \rho / 2)} u(x+y) d y \mid \\
\leqq & 2 \varepsilon / 3+|\operatorname{El}(x: 0, \rho / 2)|^{-1} \int_{E 1\left(z^{*}: 0, \rho^{\prime}\right)}\left|u\left(z^{*}+y\right)-u\left(z^{*}\right)\right| d y
\end{aligned}
$$

where $\rho^{\prime}=2 \rho K_{2}^{-2}$ and, by (3), $\mathrm{El}(x: 0, \rho / 2) \subseteq \operatorname{El}\left(z^{*}: 0, \rho^{\prime}\right)$. Thus, since $\rho^{\prime} \leqq \delta$, by (3) and (iii),

$$
\leqq 2 \varepsilon / 3+\varepsilon / 3 \text {. }
$$

Thus $Z$ is empty.

Since $u$ is a $t_{2}$ solution of (2) we have $L^{*} u \equiv L_{*} u \equiv 0$. Since $u$ is also continuous, by Lemma $3 u$ is subelliptic in $\Omega$. $-u$ can also be seen to be subelliptic and thus $u$ is a classical solution of $(2)$; the proof is complete.

4. Subelliptic functions and exceptionai sets. We shall need a few lemmas in order to prove Theorem 2. For fixed $y$ in $\Omega$ denote by $L_{y}^{\prime}$ the elliptic operator in $\Omega$ with constant coefficients $\left(a_{i j}(y)\right)$. Denote by $E(x, y)$ the singular solution of $L_{y}^{\prime} u(x)=0$ for $x$ in $\Omega-\{y\}$ of order $r^{2-K}$ at $y$, i.e. if $\mathscr{P} *\left(a_{i j}(y)\right) \mathscr{P}=\mathscr{D}=\left(\lambda_{i}(y)\right)$, then

$$
E(x, y)=\left|\mathscr{D}^{-1 / 2} \mathscr{P} *(x-y)\right|^{2-K} .
$$

Likewise we define $S(x, y)=\left|\mathscr{D}^{-1 / 2} \mathscr{P}^{*}(x-y)\right|^{2+\alpha-K}$. Thus if $a_{i j}(y)=\delta_{i j}$, then $E(x, y)=|x-y|^{2-K}$ and $S(x, y)=|x-y|^{2+\alpha-K}$. If we write

$$
L^{\prime} E(x, y)=L_{y}^{\prime} E(x, y)+\left[L_{y}^{\prime}-L^{\prime}\right] E(x, y)
$$

where $L^{\prime}$ is the principal part of $L$ and the partials are taken with respect to the first variable, a simple calculation yields, for $x \neq y,|L E(x, y)| \leqq C_{3}|x-y|^{\alpha-K}$ where $C_{3}$ is independent of $x$ and $y$. Likewise

$$
L S(x, y)=-C_{4}(x, y)|x-y|^{\alpha-K}+O\left(|x-y|^{2 \alpha-K}\right)
$$

where $C_{4}(x, y)$ is positive and bounded away from 0 and $\infty$. Let $C_{4}=\inf C_{4}(x, y)$, then for some $\eta$, if $|x-y|<\eta$

$$
L S(x, y) \leqq-\left(C_{4} / 2\right)|x-y|^{\alpha-K} .
$$


Whence for any $C_{5} \geqq 2 C_{3} C_{4}^{-1}$ and setting $G_{1}(x, y)=E(x, y)+C_{5} S(x, y), L G_{1}(x, y)$ $\leqq 0$ for $0<|x-y|<\eta$. For $|x-y|>\eta,|L E(x, y)|$ and $|L S(x, y)|$ are bounded. We can therefore find $H(x)$ so that

(1) $H(x) \geqq 0$ and

(2) $L H(x) \leqq-2 \max _{y \text { in } \Omega}\{|L E(x, y)|,|L S(x, y)|\}$, and thus for $G(x, y)=E(x, y)+C_{5} S(x, y)+C_{5} H(x)$,

$$
L G(x, y) \leqq 0 \text { for } x \neq y .
$$

From (3) we see that

$$
G(x, y)=O\left(|x-y|^{2-K}\right)
$$

independent of $x$ and $y$. $(G(x, y)$ has previously appeared in [6, p. 317].) By (15), for $f$ in $L_{1}(\Omega)$,

$$
F(x)=-\omega_{K}^{-1} \int_{\Omega} f(y) G(x, y) d y
$$

exists a.e. in $\Omega$.

LEMMA 5. Let $f$ be a nonpositive function which is bounded from below and is upper semicontinuous on $\Omega$. Form $F(x)$ as in $(16)$. Then $F(x)$ is in $C^{1}(\Omega)$ and

$$
L^{*} F(x) \leqq f(x) \text {. }
$$

(In the proof $C_{5}$ is taken a bit larger than $2 C_{3} C_{4}^{-1}$.)

Proof. This lemma is motivated by (3.7) of [11, p. 282]. The boundedness of $f$ ensures the continuity of $F(x)$. By a routine modification of the proof in [7, p. 175] that the potential due to a bounded density is in class $C^{1}$, where the partials are taken under the integral sign, we can establish the same for $F(x)$ (this is not needed if $L$ has no first order terms).

We first focus on the principal part $L^{\prime}$ of $L$ and assume that $x$ is the origin and El $(0: 0, r)=B(0, r)$. By Fubini's theorem we write

$$
\begin{aligned}
& \omega_{K} L^{\prime}(r) F(0) \\
& =-2(K+2)\left[r^{2}|B(0, r)|\right]^{-1} \int_{B(0, r)} \int_{\Omega} f(Q)\{G(M, Q)-G(0, Q)\} d Q d M \\
& =2(K+2) r^{-2} \int_{B(0, r)} f(Q)\left\{G(0, Q)-|B(0, r)|^{-1} \int_{B(0, r)} G(M, Q) d M\right\} d Q \\
& \quad+2(K+2) r^{-2} \int_{\Omega-B(0, r)} f(Q)\left\{G(0, Q)-|B(0, r)|^{-1} \int_{B(0, r)} G(M, Q) d M\right\} d Q \\
& =2(K+2) r^{-2}\left[I_{1}(r)+I_{2}(r)\right],
\end{aligned}
$$

where $r$ is sufficiently small to allow $B(0, r) \subset \Omega$.

We show first that for some $r_{0}$, if $r \leqq r_{0}$,

$$
0 \leqq G_{1}(0, Q)-|B(0, r)|^{-1} \int_{B(0, r)} G_{1}(M, Q) d M
$$


in $I_{1}(r)$, i.e., for $Q$ in $B(0, r)$, where $G_{1}(M, Q)=E(M, Q)+C_{5} S(M, Q)$. We fix $Q$ and estimate $E(M, Q)$. The estimate for $S(M, Q)$ follows in the same way.

$$
\begin{aligned}
E(0, Q)-\mid & \left.B(0, r)\right|^{-1} \int_{B(0, r)} E(M, Q) d M \\
= & \left\{E(0, Q)-\left|\mathrm{El}\left(Q: 0, r^{\prime}\right)\right|^{-1} \int_{E 1\left(Q: 0, r^{\prime}\right)} E(M, Q) d M\right\} \\
& +\left\{\left|\mathrm{El}\left(Q: 0, r^{\prime}\right)\right|^{-1} \int_{E 1\left(Q: 0, r^{\prime}\right)} E(M, Q) d M\right. \\
& \left.-|B(0, r)|^{-1} \int_{B(0, r)} E(M, Q) d M\right\} \\
= & E_{1}(r)+E_{2}(r),
\end{aligned}
$$

where $r^{\prime}$ is the smallest positive real number which allows $B(0, r) \subset \operatorname{El}\left(Q: 0, r^{\prime}\right)$. $E_{1}(r) \geqq 0$ as can be seen by a change of variables and by the superharmonicity of the singular solution of Laplace's equation. We express

$$
\begin{aligned}
E_{2}(r)= & E_{21}(r)+E_{22}(r) \\
= & |B(0, r)|^{-1} \int_{E 1\left(Q: 0, r^{\prime}\right)-B(0, r)} E(M, Q) d M+\left\{|B(0, r)|-\left|\mathrm{El}\left(Q: 0, r^{\prime}\right)\right|\right\} \\
& \cdot|B(0, r)|^{-1}\left|\mathrm{El}\left(Q: 0, r^{\prime}\right)\right|^{-1} \int_{\mathrm{El}\left(Q: 0, r^{\prime}\right)} E(M, Q) d M .
\end{aligned}
$$

$E_{21}(r) \geqq 0$ since $E(M, Q)$ is positive.

We estimate $E_{22}(r)$, which is negative. It is easy to see using Geršgorin's disks [8, p. 197] and the Hölder continuity of the $a_{i j}$ 's that

$$
\left|1-\lambda_{i}^{1 / 2}(Q)\right| \leqq\left|1-\lambda_{i}(Q)\right| \leqq C_{6}|Q|^{\alpha}
$$

where $C_{6}$ depends only on $K, K_{1}$, and $K_{2}$. If $\lambda_{1}(Q)$ is the smallest eigenvalue, then from normalizing the trace we have $r \leqq r^{\prime}=r \lambda_{1}^{-1 / 2}(Q)$; let $\lambda_{K}(Q)$ be the largest. Hence

$$
\begin{aligned}
0 & \geqq|B(0, r)|-\left|\mathrm{El}\left(Q: 0, r^{\prime}\right)\right| \geqq|B(0, r)|-\left|B\left(0, r^{\prime} \lambda_{K}^{1 / 2}(Q)\right)\right| \\
& =|B(0, r)|-\left|B\left(0, r \lambda_{1}^{-1 / 2}(Q) \lambda_{K}^{1 / 2}(Q)\right)\right|=|B(0, r)|\left(1-\lambda_{1}^{-K / 2}(Q) \lambda_{K}^{K / 2}(Q)\right)
\end{aligned}
$$

and by (20) and the above

$$
|| B(0, r)|-| \mathrm{El}\left(Q: 0, r^{\prime}\right)|| \leqq C_{7}|B(0, r)||Q|^{\alpha} \leqq C_{7}|B(0, r)| r^{\alpha}
$$

where $C_{7}$ depends only on $K, K_{1}$, and $K_{2}$. Making the appropriate replacement of the above in $E_{22}(r)$ it follows easily that

$$
\left|E_{22}(r)\right| \leqq C_{9} r^{2+\alpha-K},
$$

$C_{9}$ having the same dependence as $C_{7}$.

Treating $S(M, Q)$ in the same fashion we get

$$
0 \leqq S_{1}(r), \quad 0 \leqq S_{21}(r), \text { and }\left|S_{22}(r)\right| \leqq C_{10} r^{2+2 \alpha-K} .
$$


The claim (18) follows once we show that

$$
C_{11} r^{2+\alpha-K} \leqq S_{1}(r)
$$

where $0<C_{11}$ and depends only on $\alpha, K, K_{1}$, and $K_{2}$. For then

$$
\begin{aligned}
G_{1}(0, Q)- & |B(0, r)|^{-1} \int_{B(0, r)} G_{1}(M, Q) d M \\
& =E_{1}(r)+E_{2}(r)+C_{5} S_{1}(r)+C_{5} S_{2}(r) \geqq E_{22}(r)+C_{5} S_{1}(r)+C_{5} S_{22}(r) \\
& \geqq\left\{-C_{9}+C_{5}\left[C_{11}-C_{10} r^{\alpha}\right]\right\} r^{2+\alpha-K}
\end{aligned}
$$

using the estimates in (21), (22), and (23). Thus there is an $r_{0}$ such that, for $r \leqq r_{0}$, $C_{11}-C_{10} r^{\alpha} \geqq 2^{-1} C_{11}>0$. Since $C_{5}$ is independent of $C_{9}, C_{10}$, and $C_{11}$, for $r \leqq r_{0}$ and $C_{5} \geqq \max \left\{2 C_{3} C_{4}^{-1}, 2 C_{9} C_{11}^{-1}\right\}$ we have that (24), therefore (18), is nonnegative.

But (23) follows quite nicely by a change of variables, treating $\alpha$ as a complex variable, and using the maximum modulus principle (see the Appendix).

Since $f$ is upper semicontinuous, for $\varepsilon>0$ and $|Q| \leqq \delta(\varepsilon) \leqq r_{0}, f(Q) \leqq f(0)+\varepsilon$. So from (18) with $r \leqq \delta(\varepsilon)$

$$
\begin{aligned}
I_{1}(r) \leqq & \{f(0)+\varepsilon\} \int_{B(0, r)} G_{1}(0, Q)-|B(0, r)|^{-1} \int_{B(0, r)} G_{1}(M, Q) d M d Q \\
& +\int_{B(0, r)} f(Q)\left\{H(0)-|B(0, r)|^{-1} \int_{B(0, r)} H(M) d M\right\} d Q .
\end{aligned}
$$

Since $H(x)$ is in $C^{2}$, the product of $r^{-2}$ and the integrand in the second term is bounded in $r$ and thus the second term vanishes as $r$ tends to 0 . An easy estimate shows that

$$
\lim _{r \rightarrow 0} 2(K+2) r^{-2} \int_{B(0, r)} S(0, Q)-|B(0, r)|^{-1} \int_{B(0, r)} S(M, Q) d M d Q=0
$$

and that

$$
\lim _{r \rightarrow 0} 2(K+2 r)^{-2} \int_{B(0, r)} E(0, Q)-|B(0, r)|^{-1} \int_{B(0, r)} E(M, Q) d M d Q=\omega_{K}
$$

using the fact that

$$
2(K+2) r^{-2} \int_{B(0, r)}|Q|^{2-K}-|B(0, r)|^{-1} \int_{B(0, r)}|Q-M|^{2-K} d M d Q=\omega_{K} .
$$

Thus

$$
\limsup _{r \rightarrow 0} \omega_{K}^{-1} 2(K+2) r^{-2} I_{1}(r) \leqq f(0) .
$$

This is almost the desired conclusion; we now consider $I_{2}(r)$, which can be expressed as

$$
\int_{\Omega} f(Q) \chi_{r}(Q)\left\{G(0, Q)-|B(0, r)|^{-1} \int_{B(0, r)} G(M, Q) d M\right\} d Q
$$


where $\chi_{r}(Q)$ is the characteristic function of the set $B(0, r)^{c}$. If we formally pass to the limit under the integral sign we get

$$
\lim _{r \rightarrow 0} 2(K+2) r^{-2} I_{2}(r)=-\int_{\Omega} f(Q) \Delta G(0, Q) d Q
$$

where $\Delta G(0, Q)$ is the Laplacian of $G$ with respect to the first variable evaluated at 0 . For the moment we assume (26) since

$$
\Delta G(0, Q)=L_{Q}^{\prime} G(0, Q)+\left(\Delta-L_{Q}^{\prime}\right) G(0, Q)=\left(\Delta-L_{Q}^{\prime}\right) G(0, Q)=O\left(|Q|^{\alpha-K}\right)
$$

and the boundedness of $f$ insures the existence of the integral. Consequently from (25) and (26)

$$
\begin{array}{rl}
\limsup _{r \rightarrow 0} & L(r) F(0) \\
& \leqq f(0)-\omega_{K}^{-1} \int_{\Omega} f(Q)\left[\Delta G(0, Q)+\sum b_{i}(0) D_{i} G(0, Q)+c(0) G(0, Q)\right] d Q .
\end{array}
$$

By the hypothesis the integrand is nonnegative and thus $L^{*} F(0) \leqq f(0)$. We now justify (26) and complete the lemma. We need only show that

$$
\Delta(r) E(0, Q)=2(K+2)|B(0, r)|^{-1} r^{-2} \int_{B(0, r)} E(M, Q)-E(0, Q) d Q
$$

is $O\left(|Q|^{\alpha-K}\right)$ in order to apply the dominated convergence theorem in (26). By a change of variables and (3)

$$
\begin{aligned}
\Delta(r) E(0, Q)=(K+2)\left|\operatorname{El}\left(Q^{\prime}: 0, r\right)\right|^{-1} r^{-2} \int_{E 1\left(Q^{\prime}: 0, r\right)} & \left|M^{\prime}-Q^{\prime}\right|^{2-K} \\
& +\left|M^{\prime}+Q^{\prime}\right|^{2-K}-2\left|Q^{\prime}\right|^{2-K} d M^{\prime}
\end{aligned}
$$

where $K_{2}|Q| \leqq\left|Q^{\prime}\right| \leqq K_{2}^{-1}|Q|, r \leqq|Q|$, and $\mathrm{El}\left(Q^{\prime}: 0, r\right)$ is the inverse ellipse of $\operatorname{El}(Q: 0, r)$ with axes $\lambda_{i}^{-1 / 2}(Q)$. By the mean value theorem

$$
\begin{aligned}
\Delta(r) E(0, Q)=(K+2)\left|\operatorname{El}\left(Q^{\prime}: 0, r\right)\right|^{-1} r^{-2} \int_{\mathrm{El}\left(Q^{\prime}: 0, r\right)-B\left(0, r^{\prime}\right)}\left|M^{\prime}-Q^{\prime}\right|^{2-K} \\
+\left|M^{\prime}+Q^{\prime}\right|^{2-K}-2\left|Q^{\prime}\right|^{2-K} d M^{\prime}
\end{aligned}
$$

where $B\left(0, r^{\prime}\right)$ is the largest ball in $\mathrm{El}\left(Q^{\prime}: 0, r\right)$; thus $r^{\prime}=r \lambda_{K}^{-1 / 2}(Q), \lambda_{K}(Q)$ being again the largest eigenvalue. It can be seen by expanding the integrand in (27), $K=4$ is quite easy, that

$$
|\Delta(r) E(0, Q)| \leqq C_{12}\left|Q^{\prime}\right|^{-2}\left|\mathrm{El}\left(Q^{\prime}: 0, r\right)\right|^{-1} \int_{E 1\left(Q^{\prime}: 0, r\right)-B\left(0, r^{\prime}\right)}\left|M^{\prime}-Q^{\prime}\right|^{2-K} d M^{\prime}
$$

where $C_{12}$ depends only on $K, K_{1}$, and $K_{2}$, and by changing variables and (15)

$$
\begin{aligned}
& \leqq C_{13}|Q|^{-2}|B(0, r)|^{-1} \int_{B(0, r)-E 1\left(Q: 0, r^{\prime}\right)}|M-Q|^{2-K} d M \\
& \leqq C_{13}|Q|^{-2}|B(0, r)|^{-1} \int_{B(0, r)-B\left(0, r^{\prime \prime}\right)}|M-Q|^{2-K} d M
\end{aligned}
$$


where $r^{\prime \prime}=r^{\prime} \lambda_{1}^{1 / 2}(Q)=r \lambda_{1}^{1 / 2}(Q) \lambda_{K}^{-1 / 2}(Q)$. Since $r \leqq|Q|$, using the mean value theorem

$$
=C_{13}|Q|^{-K}\left\{1-\left|B\left(0, \lambda_{1}^{1 / 2}(Q) \lambda_{K}^{-1 / 2}(Q)\right)\right||B(0,1)|^{-1}\right\} \leqq C_{14}|Q|^{-K+\alpha}
$$

by (20); $C_{14}$ depends only on $K, K_{1}$, and $K_{2}$. Thus

$$
|\Delta(r) E(0, Q)|=O\left(|Q|^{-K+\alpha}\right)
$$

and the proof of the lemma is complete.

LEMMA 6. Let $u$ be continuous in $\Omega$ and subelliptic on $\Omega-E, E$ relatively closed and $\operatorname{Cap}(E)=0$. Then $u$ is subelliptic on $\Omega$.

Proof. This lemma follows easily using the technique in [14, p. 70] (see the Appendix).

LEMMA 7. Let $u$ be continuous in $\Omega$ and subelliptic on $\Omega-E$, where $E$ is relatively closed. Let $L^{*} u(x) \geqq 0$ everywhere on $E$. Then $u$ is subelliptic on $\Omega$.

Proof. The lemma is easily proved along the lines of Lemma 3.

Proof of Theorem 2. $E=\bigcup E_{n}$ with $E_{n}$ closed and Cap $\left(E_{n}\right)=0$. Let $A$ be the set of all points $x$ in $\Omega$ such that $u$ is not subelliptic on any neighborhood of $x$. Clearly $A$ is closed. If we show that $A$ is empty we are finished.

For positive integral $n$, let $H_{n}$ be the set of all points $x$ in $\Omega$ such that

$$
L(r) u(x) \geqq-n \text { for } 0<r<1 / n \text {. }
$$

Since $L(r) u(x)$ is continuous in $x$ for fixed $r, H_{n}$ is relatively closed. By hypothesis $\Omega-E \subset \bigcup_{n} H_{n}$. Hence $A$ is covered by $\bigcup_{n} H_{n} \cup \bigcup_{n} E_{n}$. Assuming $A$ is not empty, we can find, by the Baire category theorem, an open ball such that $A \cap B$ is not empty and $A \cap B$ is contained in one of the covering sets.

If $A \cap B \subset E_{n_{0}}$, for some $n_{0}$, then, by Lemma $6, u$ is subelliptic in $B$ and thus $A \cap B$ is empty. Thus $A \cap B \subset H_{n_{0}}$ for some $n_{0}$.

Define $f(x)=\min \left\{L_{*} u(x), 0\right\}$ for $x$ in $A \cap B \cap H_{n_{0}}$ and 0 otherwise; hence, $0 \geqq f(x) \geqq-n_{0}$ for all $x$ in $B$ and by hypothesis $f(x)=0$ a.e. in $B$. By the VitaliCarathéodory theorem there are $f_{i}$ with

(i) $-n_{0} \leqq f_{1}(x) \leqq f_{2}(x) \leqq \cdots \leqq f_{i}(x) \leqq \cdots \leqq f(x)$,

(ii) $f_{i}$ is upper semicontinuous,

(iii) $f_{i}(x) \rightarrow f(x)$ a.e. in $B$.

Form $F_{i}(x)$ as in Lemma 5 , and by Lemma $5, L^{*} F_{i}(x) \leqq f_{i}(x)$, thus $L_{*}\left(-F_{i}(x)\right)$ $\geqq-f_{i}(x) \geqq 0$. Clearly $u(x)-F_{i}(x)$ is subelliptic in $B-A$; for $x$ in $A$

$$
L^{*}\left(u(x)-F_{i}(x)\right) \geqq L_{*}\left(u(x)-F_{i}(x)\right) \geqq L_{*} u(x)+L_{*}\left(-F_{i}(x)\right) \geqq f(x)-f_{i}(x) \geqq 0 .
$$

By Lemma $7, u-F_{i}$ is subelliptic on $B$. Since $u-F_{i}$ converge monotonically to $u$, which is continuous, it is easily seen that $u$ is subelliptic on $B$ and hence $A \cap B=\varnothing$, a contradiction. The proof is complete. 
5. $t_{2}$ solutions and exceptional sets. Theorem 3 follows from Theorems 1 and 2 and the following lemma which is motivated by Lemma 6.16 [18, p. 349]. Such methods of removing dense sets have been previously employed by V. L. Shapiro in several settings as [15], [16], and [17].

LEMMA 8. Let $u$ be $a t_{2}$ solution of (2) in $\Omega-E, E$ an $F_{\sigma}$ set and $\operatorname{Cap}(E)=0$. Let $u$ be in $L_{\infty}$. In addition we assume that $\left|\Delta A_{t}(u, x)\right| \leqq M(x)$ for all $x$ in $\Omega$ and $0<t \leqq 1$, where $M(x)$ is a finite constant depending on $x$. Then $u$ is equal to a classical solution a.e. in $\Omega$.

Proof. Since $\Delta A_{t}(u, x)$ is bounded at every point of $\Omega$, we can argue as in Lemma 2 and show that $\lim _{t \rightarrow 0} A_{t}(u, x)=u_{1}(x)$ exists everywhere in $\Omega$ and converges on $\Omega-E$ to $u$. We extend $u \equiv u_{1}$ on $E$. As in Lemma 2 for each ball $B$, there is a subball $B_{1}$ on which $u$ is continuous. Let $Z$ be the nowhere dense set of discontinuities of $u$; let $\bar{Z}$ denote its closure in $\Omega$. If $\bar{Z}$ is empty, then $u$ is continuous and by hypothesis $u$ is a $t_{2}$ solution of (2) on $\Omega-E$ and therefore $L_{*} u=L^{*} u=0$ on $\Omega-E$. By Theorem $2, u$ is subelliptic in $\Omega$. Since $L^{*} u=0$ on $\Omega-E, L_{*}(-u)=0$ and $-u$ is subelliptic in $\Omega$ and consequently $u$ must be a classical solution of (2).

Assume $\bar{Z} \neq \varnothing$; then there is a ball $B_{1}$ such that $B_{1} \cap Z \neq \varnothing$ and $u$ is continuous on $\bar{B}_{1} \cap \bar{Z}$ in the topology of $\bar{B}_{1} \cap \bar{Z}$. Define

$$
H_{n}=\left\{z: z \in \bar{B}_{1} \cap \bar{Z} \text { and } U(z, r) \leqq n, 0<r \leqq 1\right\}
$$

with $U$ defined as in the proof of Theorem 1 .

Since $u$ is continuous on $\bar{B}_{1} \cap \bar{Z}, H_{n}$ is relatively closed. The union $\bigcup_{n} H_{n}$ covers $\Omega-E$ since $u$ is in $t_{2}$, therefore $t_{1}$, off $E$. So $\bigcup_{n} H_{n}$ together with $\bigcup_{n} E_{n}$ cover $\bar{B}_{1} \cap \bar{Z}$. By the Baire category theorem there is a ball $B_{2} \subset B_{1}$ such that $B_{2} \cap Z \neq \varnothing$ and for some $n_{0}$ either $\bar{B}_{2} \cap \bar{Z} \subset H_{n_{0}}$ or $\bar{B}_{2} \cap \bar{Z} \subset E_{n_{0}}$. The latter is not possible since $u$ would be a classical solution on $B_{2}-E_{n_{0}}$ by Theorem 2 , using the same argument as above when $\bar{Z}=\varnothing$, and therefore by Serrin's Theorem $1\left[14\right.$, p. 68] for $L_{\infty}$ solutions of (2), $u$ would extend to be a classical solution $v$ in $\bar{B}_{2}$. Clearly $v \equiv u_{1}$ on $E_{n_{0}} \cap \bar{B}_{2}$ and thus $B_{2} \cap Z=\varnothing$.

If $\bar{B}_{2} \cap \bar{Z} \subset H_{n_{0}}$, then $U(z, r) \leqq n_{0}$ for $z$ in $\bar{B}_{2} \cap \bar{Z}, 0<r \leqq 1$, which is (12) in Theorem 1 . The proof is now completed simply by following the proof of Theorem 1 from this point.

Proof of Theorem 3 (sufficiency). Define

$$
J_{n}=\left\{x:\left|\Delta A_{t}(u, x)\right| \leqq n, 0<t \leqq 1\right\} .
$$

$J_{n}$ is relatively closed and $\bigcup J_{n}$ is an $F_{\sigma}$ set; $G=\bigcap J_{n}^{c}$ is a $G_{\sigma}$ set and is precisely the set of points at which $\Delta A_{t}(u, x)$ is unbounded for $0<t<1$. If we show that $G$ is empty, then the theorem will follow from Lemma 8.

By 6.17 of [18, p. 349], $G$ is not a sum of nondense sets; by Lemma 1 , since $u$ is in $t_{2}$ on $\Omega-E, G$ must be contained in $E$. So $G=G \cap E=\bigcup_{i}\left(E_{i} \cap G\right)$. So for some $i_{0}$ and some open ball $B_{1}, B_{1} \cap E_{i_{0}} \cap G \neq \varnothing$ and is dense in $B_{1} \cap G$. Since $E_{i_{0}}$ is 
closed, $B_{1} \cap G=B_{1} \cap E_{i_{0}} \cap G$. Again by Lemma 8, $u$ is a classical solution of (2) on $B_{1}-E_{i_{0}}$, thus on $B_{1}$. So $B_{1} \cap G=\varnothing$, which is impossible. The proof of the sufficiency is complete.

Proof of Theorem 3 (necessity). We shall assume that $\Omega$ is a Dirichlet domain or more simply that $\Omega=B(0,1)$. The following remarks are used to establish the necessity:

REMARK 1. If $L u_{n}=0$ and $u_{n}$ converge uniformly to $u$ in $\Omega$, then $L u=0$.

Proof. Let $B$ be an open ball with $\bar{B} \subset \Omega$. Since $u$ is continuous on $\bar{B}$, let $v$ be the solution of $L v=0, v \equiv u$ on $\partial B$. Then for $n$ sufficiently large

$$
-\varepsilon \leqq v-u_{n} \leqq \varepsilon \quad \text { on } \partial B
$$

and therefore in $B$ by the maximum principle. So $v \equiv u$ in $B$.

REMARK 2. If $L u_{n}=0$ and $u_{n} \leqq u_{n+1}$, then, for $u=\lim _{n \rightarrow \infty} u_{n}, L u=0$ provided that $\left\|u_{n}\right\|_{\infty}$ are uniformly bounded by $M<\infty$.

Proof. If $u$ is continuous, then the $u_{n}$ converge uniformly to $u$ by Dini's theorem and, by Remark $1, L u=0$. Fix $x_{0}$ in $\Omega$ and let $\varepsilon>0$ so that $B\left(x_{0}, 4 \varepsilon K_{2}^{-1}\right) \subset \Omega$. Denote by ${ }_{r} u(x)$ the average of $u$ over the ellipse El $(x: x, r)$. We can choose $\delta$ so that $\delta \leqq \varepsilon$ and

$$
\left|{ }_{\varepsilon} u(x)-{ }_{\varepsilon} u\left(x_{0}\right)\right|<\varepsilon \text { for }\left|x-x_{0}\right|<\delta
$$

For $\left|x-x_{0}\right|<\delta$,

$$
\begin{aligned}
\left|u(x)-u\left(x_{0}\right)\right| & \leqq\left|u(x)-{ }_{\varepsilon} u(x)\right|+\left|u\left(x_{0}\right)-{ }_{\varepsilon} u\left(x_{0}\right)\right|+\left|{ }_{\varepsilon} u(x)-{ }_{\varepsilon} u\left(x_{0}\right)\right| \\
& \leqq \lim _{n \rightarrow \infty}\left|u_{n}(x)-{ }_{\varepsilon} u_{n}(x)\right|+\lim _{n \rightarrow \infty}\left|u_{n}\left(x_{0}\right)-{ }_{\varepsilon} u_{n}\left(x_{0}\right)\right|+\varepsilon
\end{aligned}
$$

by (28) and the dominated convergence theorem. Since $\mathrm{El}\left(x_{0}: x_{0}, 2 \varepsilon\right)$ and $\mathrm{El}(x: x, 2 \varepsilon)$ are contained in $\Omega$, by Lemma 4 the above is $\leqq 2 C_{2} M \varepsilon^{\alpha}+\varepsilon$. Hence $u$ is continuous in $\Omega$ and the proof is complete. Let $G(x, y)$ be defined as in Lemma 5 and define $J(x, y)=E(x, y)-C_{5}(S(x, y)+H(x))$.

REMARK 3. $L G(x, y) \leqq 0$ and $L J(x, y) \geqq 0$ for $x$ in $\Omega-\{y\}$ and each $y$ in $\Omega$.

Proof. $L G(x, y) \leqq 0$ has been established. Using the estimates leading to (14) for $|x-y|<\eta$

$$
\begin{aligned}
L E(x, y)-C_{5} L S(x, y)-C_{5} L H(x) & \geqq L E(x, y)-C_{5} L S(x, y) \\
& \geqq-C_{3}+\left(C_{5} C_{4} / 2\right)|x-y|^{\alpha-K} \geqq 0 .
\end{aligned}
$$

For $|x-y| \geqq \eta$ it is also obvious.

REMARK 4. $J(x, y) \leqq-1$ for $y$ in $B\left(0, \frac{1}{2}\right)$ and $x$ in $B\left(0, \frac{3}{4}\right)^{c}$.

Proof. Both $S(x, y)$ and $H(x)$ are positive and we can choose $C_{5}$ as large as we wish.

REMARK 5. $0<J(x, y)$ for $|x-y|<\gamma$ where $\gamma$ depends on $\alpha, K_{2}$, and $C_{5}$.

Proof. $E(x, y)$ is positive and dominates $S(x, y)$ and $H(x)$ when $|x-y|$ is small. 
Assume that $E$ is closed, Cap $(E)>0$, and $E \subset B\left(0, \frac{1}{2}\right)$; in addition we can assume without loss of generality that the diameter of $E$ is less than $\gamma / 2$. Let $\lambda$ be the equilibrium distribution of $E$ and form

$$
u_{\lambda}(x)=\int_{E} J(x, y) d \lambda(y), \quad v_{\lambda}(x)=\int_{E} G(x, y) d \lambda(y) .
$$

Since $\int_{E}|x-y|^{2-K} d \lambda(y)$ is bounded in $\Omega-E$, by (15) and the definition of $J(x, y)$, $u_{\lambda}$ and $v_{\lambda}$ are bounded. By Remark 3 and Lemma $3, u_{\lambda}$ is subelliptic and $v_{\lambda}$ is superelliptic in $\Omega-E$, and $u_{\lambda}<v_{\lambda}$ since $J(x, y)<G(x, y)$.

We define

$$
\begin{aligned}
\mathscr{M}=\{v: v & \in C(\Omega-E), v \equiv u_{\lambda} \text { on } \partial \Omega, \\
v & \left.\leqq v_{\lambda} \text { in } \Omega-E, v \text { is subelliptic in } \Omega-E\right\} ;
\end{aligned}
$$

$\mathscr{M} \neq \varnothing$ since $u_{\lambda} \in \mathscr{M}$. Furthermore it is easy to see that

(i) if $v_{1}, v_{2} \in \mathscr{M}$, then $\sup \left(v_{1}, v_{2}\right) \in \mathscr{M}$;

(ii) if $\bar{B}$ is any closed ball in $\Omega-E$ and $v \in \mathscr{M}$, then the continuous function $V=v$ off $B$ and, satisfying $L V=0$ in $B$, is in $\mathscr{M}$.

That $v_{\lambda}$ is superelliptic is needed to establish (ii). Our desired function is $u(x)$ $=\sup _{v \in \mathscr{M}} v(x)$. Clearly $u_{\lambda}(x) \leqq u(x) \leqq v_{\lambda}(x)$. By a slight modification of the standard argument in the Perron method it is easy to show, using Remark 2 together with (i) and (ii), that $L u(x)=0$ in $\Omega-E$; such a modification is not needed if $c(x) \equiv 0$. Thus $u$ is bounded and $L u=0$. We need only prove that $u$ does not extend to be a solution of (2) on $E$.

By Remark $5, u_{\lambda}$ is positive if the distance from $x$ to $E$ is less than $\gamma / 2$; so $u$ must be positive for dist $(x, E)<\gamma / 2$. If $u$ is nonpositive near $\partial B(0,1)$, then $u$ cannot possibly be a solution in $B(0,1)$ by the maximum principle. Without loss of generality we can assume that $G_{1}(x, 0)=|x|^{2-K}+C_{5}|x|^{2+\alpha-K}$. Since $B(0,1)$ is a Dirichlet domain we can also assume that $H(x)$ vanishes on $\partial B(0,1)$; set $g(x) \equiv G_{1}(x, 0)$ on $\partial B(0,1)$ with $L g(x)=0$. Since $g(x)$ takes its maximum on the boundary, $g(x)$ $<G_{1}(x, 0)$ for $|x|<1$, and $g(x)<G_{1}(x, 0)+C_{5} H(x)$, for $H(x)$ is nonnegative. So $G(x, 0)-g(x)$ vanishes on $\partial B(0,1)$, is strictly positive in the interior, and is superelliptic. For a suitably large constant $C_{15}$ and for $x$ on $\partial B\left(0, \frac{1}{2}\right)$

$$
u(x) \leqq C_{15}[G(x, 0)-g(x)]=\mathscr{G}(x) .
$$

By Remark 4 every element of $\mathscr{M}$ is majorized by $\mathscr{G}(x)$ on $\partial B(0,1)$ and is majorized on $\partial B\left(0, \frac{1}{2}\right)$ by $u$ and therefore by $\mathscr{G} . \mathscr{G}$ is superelliptic so every element in $\mathscr{M}$ is majorized on $B(0,1)-B\left(0, \frac{1}{2}\right)$. Thus $u$ is also majorized; its positive maximum cannot occur on $\partial B(0,1)$ if it is a solution in $B(0,1)$.

Appendix. We now establish (23). $Q$ is fixed and $|Q| \leqq r$.

$$
S_{1}(r)=S(0, Q)-\left|\mathrm{El}\left(Q: 0, r^{1}\right)\right|^{-1} \int_{\mathrm{El}\left(Q: 0, r^{\prime}\right)} S(M, Q) d M
$$


where $r \leqq r^{\prime}=r \lambda_{1}^{-1 / 2}(Q)$. By a change of variables, the above is equal to

$$
\left|Q^{\prime}\right|^{2+\alpha-K}-\left|B\left(0, r^{\prime}\right)\right|^{-1} \int_{B\left(0, r^{\prime}\right)}\left|M^{\prime}-Q^{\prime}\right|^{2+\alpha-K} d M^{\prime},
$$

where $\left|Q^{\prime}\right| \leqq r^{\prime}$, which in turn is equal to

$$
r^{2+\alpha-K}\left\{\left|Q^{\prime \prime}\right|^{2+\alpha-K}-|B(0,1)|^{-1} \int_{B(0,1)}\left|M^{\prime \prime}-Q^{\prime \prime}\right|^{2+\alpha-K} d M^{\prime \prime}\right\}
$$

where $\left|Q^{\prime \prime}\right| \leqq 1$. If the term in braces is larger than a positive constant $C_{11}^{\prime}$ which depends only on $\alpha, 0<\alpha \leqq 1$, then clearly $C_{11}^{\prime} r^{2+\alpha-K} \leqq C_{11} r^{2+\alpha-K}$ where $C_{11}$ is positive and depends only on $C_{11}^{\prime}, K, K_{1}$, and $K_{2}$. The expression in braces is equal to

$$
1-\left|B\left(0,\left|Q^{\prime \prime}\right|^{-1}\right)\right|^{-1} \int_{B\left(0,\left|Q^{\prime \prime}\right|-1\right)}\left|M^{m}-Q^{m}\right|^{2+\alpha-K} d M^{m}
$$

where $\left|Q^{m}\right|=1 \leqq\left|Q^{\prime \prime}\right|^{-1}$. We consider

$$
\left|B\left(0,\left|Q^{\prime \prime}\right|^{-1}\right)\right|^{-1} \int_{B\left(0,\left|Q^{\prime \prime}\right|-1\right)}\left|M^{\prime \prime}-Q^{m}\right|^{2+\alpha-B} d M^{m}=f(\alpha)
$$

as a function of the complex variable $\alpha$ with domain $0 \leqq \operatorname{Re} \alpha \leqq K-2,-1 \leqq \operatorname{Im} \alpha \leqq 1$. It is a routine matter to show that $f(\alpha)$ is analytic.

Setting $\alpha=x+i y$ we observe that $|f(x+i y)| \leqq f(x)$, hence $f$ takes a maximum modulus either at $\alpha=0$ or at $\alpha=K-2$. When $\alpha=K-2, f(\alpha)=1$; when $\alpha=0$, $0 \leqq f(\alpha) \leqq\left|Q^{m}\right|^{2-K}=1$ by the superharmonicity of the singular solution of Laplace's equation. Thus if $f$ is nonconstant, for positive $\alpha$ we have that $f(\alpha)<1$. But $f$ is nonconstant if we extend its domain to $-2<\operatorname{Re} \alpha$.

We fix $\alpha$ in $0<\alpha \leqq 1$ and vary $\left|Q^{\prime \prime}\right|$ to show that (28) is strictly positive for $\left|Q^{\prime \prime}\right| \leqq 1$. But it is obvious since (28) tends to 1 as $\left|Q^{\prime \prime}\right|$ tends to 0 . Thus (28) is greater than some positive constant $C_{11}^{\prime}$ which depends on $\alpha$.

Proof of Lemma 6. We use the technique in [14, p. 70]. Since $u$ is continuous we can solve $L V=0$ in $B$ and $v=u$ on $\partial B$ where $\bar{B}$ is any fixed ball in $\Omega$. We need only show that $u \leqq v$ in $B$.

Let $x$ be any point in $B-B \cap E$. Since $\operatorname{Cap}(E)=0, \operatorname{Cap}(\bar{B} \cap E)=0$. Let $\mathcal{O}_{1}, \mathcal{O}_{2}, \ldots$ be a nested sequence of closed sets with smooth boundaries, converging to $\bar{B} \cap E$, such that $\operatorname{Cap}\left(\mathcal{O}_{i}\right)$ tends to 0 . Denote by $\mu_{i}$ the equilibrium distribution for $\mathcal{O}_{i} ;$ it is well known that

$$
\Phi_{i}(x)=\int_{\mathcal{O}_{i}}|x-y|^{2-K} d \mu_{i}(y) \cdot I\left(\mu_{i}\right)^{-1}
$$

is identically 1 on $\partial \mathcal{O}_{i}$, where $I\left(\mu_{i}\right)$ is the energy integral and $I\left(\mu_{i}\right) \rightarrow \infty$ as $i \rightarrow \infty$ since $\operatorname{Cap}\left(\mathcal{O}_{i}\right) \rightarrow 0$. Form

$$
\Psi_{i}(x)=\int_{\mathcal{O}_{i}} G(x, y) d \mu_{i}(y) \cdot I\left(\mu_{i}\right)^{-1}
$$


with $G$ as in Lemma 5 . Clearly $\Psi_{i}$ is superelliptic on $\Omega-\mathcal{O}_{i}$ and is bounded away from 0 on $\partial \mathcal{O}_{i}$ by a constant $M_{1}$ independent of $i$. If $M_{0}$ is the maximum of $u-v$ in $\bar{B}$ then $u-v \leqq M_{0} M_{1}^{-1} \Psi_{i}$ on $\partial \mathcal{O}_{i}$ and $\partial B$ and thus in $B-\mathcal{O}_{i}$. So letting $i \rightarrow \infty$, $u(x)-v(x) \leqq 0$ for $x$ in $B-B \cap E$, thus, a.e. in $\bar{B}, u$ and $v$ are continuous, so $u \leqq v$ in $\bar{B}$.

The following simple example shows that the $t_{\alpha}$ notion cannot be replaced by the generalized elliptic operators $L^{*}$ or $L_{*}$ in Theorems 1 and 3. It also shows that the continuity hypothesis cannot be simply eliminated in Theorem 2 .

Define $u(x)$ to be 1 in open upper half space, $0<x_{1},-1$ in open lower half space, and 0 for $x_{1}=0$. Then for each $x$ in $E_{K}$

$$
\lim _{r \rightarrow 0} 2(K+2)|B(0, r)|^{-1} r^{-2} \int_{B(0, r)} u(x+y)-u(x) d y=0
$$

so that $\Delta^{*} u(x)=\Delta_{*} u(x)=0 . u$ is clearly not harmonic in $E_{K}$.

\section{BIBLIOGRAPHY}

1. M. G. Arsove, The Looman-Menchoff theorem and some subharmonic function analogues, Proc. Amer. Math. Soc. 6 (1955), 94-105. MR 16, 1108.

2. A. P. Calderón and A. Zygmund, Local properties of solutions of elliptic partial differential equations, Studia Math. 20 (1961), 171-225. MR 25 \#310.

3. L. Carleson, Selected problems on exceptional sets, Van Nostrand Math. Studies, no. 13, Van Nostrand, Princeton, N. J., 1967. MR 37 \#1576.

4. R. Courant and D. Hilbert, Methods of mathematical physics. Vol. II: Partial differential equations, Interscience, New York, 1962. MR 25 \#4216.

5. A. Douglis and L. Nirenberg, Interior estimates for elliptic systems of partial differential equations, Comm. Pure Appl. Math. 8 (1955), 503-538. MR 17, 743.

6. D. Gilbarg and J. Serrin, On isolated singularities of solutions of second order elliptic differential equations, J. Analyse Math. 4 (1955/56), 309-340. MR 18, 399.

7. G. Hellwig, Partial differential equations: An introduction, Blaisdell, New York, 1964. MR 30 \#3286.

8. M. Marcus and H. Minc, Introduction to linear algebra, Macmillan, New York; CollierMacmillan, London, 1965. MR 32 \#5660.

9. S. P. Philipp, Hankel transforms and generalized axially symmetric potentials, Thesis, University of California, Riverside, Calif., 1969.

10. T. Radó, Subharmonic functions, Chelsea, New York, 1949.

11. W. Rudin, Integral representations of continuous functions, Trans. Amer. Math. Soc. 68 (1950), 278-286. MR 11, 663.

12. —-, Real and complex analysis, McGraw-Hill, New York, 1966. MR 35 \#1420.

13. S. Saks, Théorie de l'intégrale, Monografie Matematyczne, vol. II, PWN, Warsaw, 1933; English transl., Monografie Matematyczne, vol. VII, PWN, Warsaw, 1937; 2nd rev. ed., Dover, New York, 1964. MR 29 \#4850.

14. J. Serrin, Removable singularities of solutions of elliptic equations, Arch. Rational Mech. Anal. 17 (1964), 67-78. MR 30 \#336. 
15. V. L. Shapiro, Fourier series in several variables, Bull. Amer. Math. Soc. 70 (1964), 48-93. MR 28 \#1448.

16. - Sets of uniqueness for the vibrating string problem, Trans. Amer. Math. Soc. 141 (1969), 127-146. MR 39 \#5961.

17. — Removable sets for pointwise solutions of the generalized Cauchy-Riemann equations, Ann. of Math. (2) 92 (1971), 82-101.

18. A. Zygmund, Trigonometric series. Vols. I, II, 2nd. ed., Cambridge Univ. Press, New York, 1968. MR 38 \#4882.

Department of Mathematics, University of California, Riverside, California 92502

Department of Mathematics, University of California, Davis, California 95616 